\title{
alpha-1 giardin is an annexin with highly unusual calcium-regulated mechanisms
}

Saroja K. Weeratunga ${ }^{1}$, Asiah Osman ${ }^{1, \#}$, Nien-Jen $\mathrm{Hu}^{2}$, Conan Wang ${ }^{1, \$}$, Lyndel Mason ${ }^{1}$, Staffan Svärd $^{3}$, Greg Hope ${ }^{4}$, Malcolm K. Jones ${ }^{5,6} \&$ Andreas Hofmann ${ }^{1,7,8, *}$

${ }^{1}$ Structural Chemistry Program, Eskitis Institute for Cell and Molecular Therapies, Griffith University, Brisbane, Qld 4111, Australia

${ }^{2}$ Membrane Protein Crystallography, Imperial College, London

${ }^{3}$ Department of Cell and Molecular Biology, Uppsala University, Sweden

${ }^{4}$ School of Biomolecular and Physical Sciences, Griffith University, Brisbane, Qld 4111, Australia

${ }^{5}$ School of Veterinary Sciences, The University of Queensland, Gatton, Qld, 4343, Australia

${ }^{6}$ Queensland Institute of Medical Research, Brisbane, Queensland 4029, Australia

${ }^{7}$ Faculty of Veterinary Science, The University of Melbourne, Parkville, Victoria 3010, Australia

${ }^{8}$ Queensland Tropical Health Alliance

*Corresponding author: a.hofmann@griffith.edu.au

\#Present address: Drug Discovery Centre

Forest Research Institute Malaysia (FRIM) Kepong,

52109 Selangor DE, Malaysia

\$Present address: Institute of Molecular Biosciences

University of Queensland, St Lucia, Qld, Australia 


\section{Abstract}

Alpha-giardins constitute the annexin proteome (group E annexins) in the intestinal protozoan parasite Giardia, and as such represent the evolutionary oldest eukaryotic annexins. The dominance of alpha-giardins in the cytoskeleton of Giardia with its greatly reduced actin content emphasises the importance of the alpha-giardins for the structural integrity of the parasite, which is particularly critical in transformation stage between cyst and trophozoite.

In this study, we report the crystal structures of the apo and calcium-bound forms of $\alpha 1$-giardin, a protein localised to the plasma membrane of Giardia trophozoites that has recently been identified as a vaccine target. The calcium-bound crystal structure of $\alpha 1$-giardin revealed the presence of a type III site in the first repeat as known from other annexin structures, as well as a novel calcium binding site situated between repeats I and IV. By means of comparison, the crystal structures of three different alpha-giardins known to date indicate that these proteins engage different calcium coordination schemes, among each other, as well as compared to annexins of groups A-D.

Evaluation of the calcium-dependent binding to acidic phosphoplipid membranes revealed that this process is not only mediated but also regulated by the environmental calcium concentration. Uniquely within the large family of annexins, $\alpha 1$-giardin disengages from the phospholipid membrane at high calcium concentrations possibly due to formation of a dimeric species. The observed behaviour is in line with changing calcium levels experienced by the parasite during excystation and may thus provide first insights into the molecular mechanisms underpinning the transformation and survival of the parasite in the host.

\section{Highlights}

- $\alpha 1$-giardin is a cytoskeletal protein of crucial importance for structural integrity of Giardia.

- The crystal structure of a1-giardin in the apo and calcium-bound forms has been determined, 
and its membrane-binding behaviour shows atypical mediation and regulation by calcium ions.

- $\alpha 1$-giardin dislocation during excystation may be triggered by calcium levels in the stomach of the host.

- This is the first time such a membrane binding mechanism has been observed with an annexin protein.

\section{Keywords}

giardiasis; host-pathogen interaction; protein-membrane interactions

\section{Introduction}

The intestinal parasite Giardia has a worldwide distribution ${ }^{1}$ and is a major cause of human protozoan-induced diarrhea. Collectively, diarrheal diseases are the second largest threat in terms of mortality and morbidity worldwide ${ }^{2}$. Giardia cysts can survive water filtration and chlorination, and the current drug treatments employ the antibiotics metronidazole, albendazole or nitazoxanide ${ }^{3}$. In common with many other infectious pathogens, Giardia has developed resistance against many drugs used to treat it $^{3}$.

Giardia possesses a complex microtubule-based cytoskeleton that is considered the main component of the parasite's virulence, due to the critical importance for its motility, attachment, intracellular transport, cell division and lifecycle transformations. The cytoskeletal components comprise structures commonly found in flagellated protists, as well as organelles unique to Giardia, such as the ventral adhesion disk, median body, funis and axoneme-associated elements ${ }^{4}$. At the protein level, the Giardia cytoskeleton consists of tubulin as well as three different giardin families, 
the alpha-giardins (annexin homologues) ${ }^{56}$, beta-giardins (homologues of striated fibre assemblin) ${ }^{7}$ and gamma-giardins (no identified homologue) ${ }^{8}$.

The ubiquitous family of annexin proteins is widespread throughout the Eukaryota, and has recently become apparent in molecular studies of diverse parasite groups. Parasite annexins, particularly those of the neodermatan platyhelminths Taenia and Schistosoma, have attracted attention due to their immunological properties or surface location (for a recent review see ${ }^{9}$ ). In the diplomonad protozoan parasite Giardia intestinalis, the peculiar family of alpha-giardins constitutes the annexin proteome. Some 21 different alpha-giardins have been identified in the genome of iardia $^{10}$. Alpha-giardins are numbered $\alpha 1-\alpha 19$, with $\alpha 7$ existing in three variants: $\alpha 7.1, \alpha 7.2$ and $\alpha 7.3$.

Since Giardia possesses a reduced actin system alpha-giardins are believed to stabilise the parasite against membrane breakdown and structural collapse in its hostile enteric environment due to their localisation at the edges of the microribbons in the ventral adhesive disk ${ }^{11}$. The ventral disk is an organelle used by the parasite for attachment to the intestinal mucosa, and is assembled from stored fragments during the process of excystation ${ }^{4}$. The transformations during disk synthesis require significant structural changes at which point individual alpha-giardins may become exposed to the host. The immunodominance of particular alpha-giardins supports this hypothesis ${ }^{12}$. Notably, different adhesion mechanisms may be employed by Giardia when comparing the excyzoite and trophozoite. While the trophozoite can rely on the ventral adhesive disk for attachment ${ }^{13}$, this organelle is not yet present at the stage of the excyzoite.

Interactions with host proteoglycans are a major component of the infection strategy of many viruses and parasites, and may therefore offer an alternative attachment mechanism at that stage ${ }^{14}$. A critical step in the invasion and subsequent cytolysis of the intestinal epithelium by parasites is the binding to surface carbohydrates of the host. Examples of glycosaminoglycan-binding proteins from pathogens in this context include the influenza haemagglutinin ${ }^{15}$, PfEMP-1 from Plasmodium falciparum $^{16}$ and the adhesins from Shiga toxin and Entamoeba histolytica ${ }^{17}$. Intruigingly, several 
annexins ( $\mathrm{A} 4^{18}, \mathrm{~A} 5^{19}, \mathrm{~A} 6^{20}$ ), including $\alpha 1$-giardin ${ }^{21}$ and annexin $\mathrm{B} 1$ from pork tape worm ${ }^{22}$, possess lectin properties, thus making them likely players in the attachment stage of infection.

So far, the crystal structures and some initial biochemical characterisation of $\alpha 11^{23}$ and $\alpha 14$ giardin ${ }^{24}$ have been reported. The crystal structure of $\alpha 11$-giardin, a protein devoid of all canonical calcium binding sites, revealed a single calcium ion bound in the first repeat. It has also been reported to lack any calcium-dependent membrane binding activity; however, only membranes with low acidic phospholipid content have been tested ${ }^{23} . \alpha 14$-giardin, mainly found in the flagella, was characterised with five bound calcium ions and its membrane binding profile includes calciumdependent binding to phosphatidylserine, phosphatidylinositol-4-phosphate and cardiolipin ${ }^{24}$.

Probably the best established alpha-giardin with potential as a vaccine candidate is $\alpha 1$-giardin which is associated with the plasma membrane of the trophozoite form of the parasite ${ }^{21}$. The protein is highly immunogenic, is a tool for immunodiagnosis ${ }^{21}$, and has also been shown to be a vaccine candidate $^{25}$. From structure-based amino acid sequence alignments it is obvious that $\alpha 1$-giardin constitutes one of the shortest alpha-giardins and consists of the annexin core domain only, distinguishing it from $\alpha 11$ - and $\alpha 14$-giardin, which have $\mathrm{N}$ - and/or C-terminal extensions. Interestingly, $\alpha 1$ - and $\alpha 2$-giardin share $77 \%$ identity at amino acid sequence level, and appear to form a pair of highly structurally related alpha-giardins.

In the current study, we report the three-dimensional crystal structures of apo and calcium-bound $\alpha 1$-giardin, as well as its unusual calcium-mediated and -regulated membrane binding behaviour. We present evidence that this behaviour is due to calcium-dependent oligomerisation and develop a model with functional implications for establishment in the host by Giardia. 


\section{Results}

\section{Crystal structure of $\alpha 1$-giardin}

The crystal structures of $\alpha 1$-giardin in the apo and calcium-bound forms were determined in the orthorhombic space group $\mathrm{P} 2{ }_{1} 2_{1} 2_{1}$. Compared to other members of the family, $\alpha 1$-giardin is a "short" annexin with no N-terminal domain (see Figure 1a). The core domain consists of the well known fourfold cyclic arrangement of five-helix bundles with repeats I/IV, and II/III each forming a module. Intra-modular interactions between the individual repeats are mainly of hydrophobic nature, whereas inter-modular interactions comprise mainly polar and ionic interactions. Each individual repeat is composed of two parallel helix-loop-helix motifs ( $\mathrm{AB}$ and $\mathrm{DE}$ ), and the fifth helix (C) is situated perpendicular to the parallel bundle on the side distal to the loops. The convex side of the molecule harbouring the exposed loops is the canonical membrane binding site of the annexins. Although the structure of $\alpha 1$-giardin contains the highly conserved annexin fold, which consists of four repeats in the core domain, there are large degrees of freedom with respect to the relative orientation of individual helices as well as the structures of loops on the convex surface compared to known annexin structures. The differences in orientation of individual $\alpha$-helices explain the frequent difficulties with molecular replacement attempts to solve novel annexin structures.

Superposition of the two structures of $\alpha 1$-giardin with those of $\alpha 11$ - and $\alpha 14$-giardin (PDB ID: 2II2, PDB ID: 3CHJ) reveals the most prominent variations in the IAB-, IIIAB- and IVDE-loop areas. $\alpha 1$-giardin possesses the shortest linkages among the three proteins in those loop areas, therefore making possible coordination of calcium ions in those loops very unlikely.

On the convex surface, the putative membrane binding side of the molecule, several prominent basic residues are located in all of the four repeats (I: Lys55, Lys56, Lys59; II: Arg89, Lys128, Lys133; III: Lys169, Lys211; IV: Lys242, Lys282). Many of those residues are conserved among the $21 \alpha$-giardins. 
In the orthorhombic crystals obtained in the presence of $15 \mathrm{mM}$ calcium, two calcium ions are bound in the I/IV module of $\alpha 1$-giardin. The apo- and calcium-bound crystal structures show no significant conformational differences, resulting in a low rms difference of $0.37 \AA$. One of the calcium ions (Ca300), corresponding to Ca505 in the structure of $\alpha 14$-giardin, occupies the type III site in the DE-loop of first repeat, and is coordinated in a tetragonal-bipyramidal geometry by the carboxylate of Glu64 (mono-dentate), the backbone carbonyl groups of Lys55 and Leu58, as well as three water molecules (see Figure $1 \mathrm{~b}$ and Supplementary Figure S1a). The other calcium ion (Ca301) occupies a novel site, previously not observed in any annexin structure. The site is located on the convex surface between helices IE, IVA and IVE, and the ion is coordinated in a tetragonalpyramidal fashion by the carboxylate groups of Glu63 (mono-dentate), Glu245 (bi-dentate) and Asp281 (mono-dentate), as well as two water molecules (see Figure 1c and Supplementary Figure S1b). When superimposing the calcium-bound structure of $\alpha 14$-giardin (PDB ID: 3CHK) with that of $\alpha 1$-giardin, it becomes obvious that apart from the calcium ion in the type III site in the first repeat, only occupation of the calcium site in the AB-loop of the second repeat (Ca503) may be possible; all other sites are absent due to steric reasons.

\section{Membrane binding}

The calcium-dependent binding of $\alpha 1$-giardin to phospholipid membranes was assessed in a liposome co-pelleting assay, using two different membrane compositions, phosphatidylserine/phosphatidylcholine (3:1) and phosphatidylserine/phosphatidylcholine (1:3). As expected, the fraction of protein bound to membranes with high phosphatidylserine content is higher than to membranes with low phosphatidylserine content (see Figure 2 and Supplementary Figure S2). Using liposomes composed of phosphatidylserine/phosphatidylcholine (3:1), from 0 to $5 \mathrm{mM}$ calcium, a typical binding curve with no apparent cooperativity is observed, and the maximal fraction of membrane-bound is about $90 \%$. Surprisingly, from about $5 \mathrm{mM}$ calcium onwards, the 
fraction of membrane-bound protein decreases continuously, reaching the low fraction of $40 \%$ membrane-bound protein at the last tested calcium concentration of $20 \mathrm{mM}$. This very behaviour is mirrored with membranes of only 25\% phosphatidylserine content. Notably, the observed binding profile is novel and is not an effect of the calcium concentrations used, as other annexins subjected to the same assay conditions yielded conventional saturation profiles ${ }^{22}$.

\section{Solution structure}

Thermal denaturation studies of $\alpha 1$-giardin monitored by circular dichroism spectroscopy in the absence and presence of $5 \mathrm{mM} \mathrm{CaCl}_{2}$ were analysed with respect to the half-point of unfolding, $\mathrm{T}_{1 / 2}$. In the presence of calcium, the protein shows an increase in $T_{1 / 2}$ of $3.5 \mathrm{~K}$, indicating that bound calcium stabilises the protein against unfolding (see Figure 3). Interestingly, the unfolding curve of $\alpha 1$-giardin in the absence of calcium shows a minor transition at around $32.1^{\circ} \mathrm{C}$. We assume that this transition reflects the disassembly of a dimeric species into monomers (see below).

Size exclusion chromatography yields different elution profiles in the presence and absence of calcium (see Figure 4 and Supplementary Table S1 for detailed analysis). In the absence of calcium, the protein elutes at 25.2 min which corresponds to a molecular mass of about $57 \mathrm{kDa}$, roughly two times the mass of monomeric $\alpha 1$-giardin. In the presence of $5 \mathrm{mM}$ calcium, the main elution peak appears at 28.1 min which corresponds to a molecular mass of about $30 \mathrm{kDa}$, i.e. monomeric $\alpha 1$ giardin. Finally, at $10 \mathrm{mM}$ calcium, two elution peaks are observed, one at $24.7 \mathrm{~min}$ and one at 28.1 min, indicating the presence of a monomeric and a dimeric species. Notably, the dimeric species observed in the absence and presence of $10 \mathrm{mM}$ calcium possess different retention times, indicating that these are two different species.

The calcium-dependent oligomerisation state of $\alpha 1$-giardin is further supported by results from small-angle X-ray scattering. Scattering data acquired at calcium concentrations ranging from 0 to $10 \mathrm{mM}$ were analysed to obtain the intensity of forward scattering, $I(0)$, and the radius of gyration, 
$R_{g}$ (see Table 2). From $I(0)$, the mass of the scattering particle can be evaluated which yields the dimeric species in the absence of calcium, a monomeric species in the presence of $0.1 \mathrm{mM}$ calcium, and a mass of about 1.3 times of monomeric $\alpha 1$-giardin in the presence of $10 \mathrm{mM}$ calcium (see Supplementary Table S2). From the size exclusion chromatograms, the presence of a significant amount of monomeric protein is obvious at the calcium concentration of $10 \mathrm{mM}$. Being nonmonodisperse, this set of samples is therefore not ideal for analysis by SAXS and a concentrationdependent trend is apparent in the $I(0)$ values (see Supplementary Table S2). We assume that these non-ideal conditions cause the estimate of about 1.3 times the mass of monomeric $\alpha 1$-giardin in case of the $10 \mathrm{mM}$ calcium dataset rather than a value closer to 2 .

The radius of gyration, $R_{g}$ is a measure of the size of the scattering particle, and the results mirror the effects seen with the molecular mass. In the absence of calcium, a species with $R_{g}$ of $36.2 \AA$ is observed, and in the presence of $0.1 \mathrm{mM}$ calcium, the experimental $R_{g}$ is $30.5 \AA$. At $10 \mathrm{mM}$ calcium, the apparent $R_{g}$ of the scattering species is $33.3 \AA$. While there is some variation between the experimental and calculated $\mathrm{R}_{\mathrm{g}}$ or $\mathrm{R}_{\text {hydro }}$ values for the monomer from the crystal structure and the calcium-bound dimer model shown in Figure 6, there is clear agreement in their relations, showing an increase from the monomer to the dimer (see Table 2).

\section{Glycosaminoglycan binding}

Alpha-giardins as well as other annexins have been reported to possess lectin properties ${ }^{192122}$. In order to evaluate the binding characteristics of $\alpha 1$-giardin to a representative glycosaminoglycan, a calcium-dependent binding assay using Heparin Sepharose was performed (see Figure 5 and Supplementary Figure S3). The protein binds to the resin in a calcium-dependent and cooperative manner ( $\mathrm{n}=1.8)$, up to a maximum fraction of $76 \%$. The half-maximum calcium concentration $\mathrm{c}_{1 / 2}$ is $0.37 \mathrm{mM}$. Notably, $\alpha 1$-giardin also displays calcium-independent binding to Heparin Sepharose with a binding fraction of $20 \%$. No irreversible binding of the protein to Heparin Sepharose was 
observed (data not shown).

\section{Discussion}

\section{Calcium binding sites in alpha-giardins}

A hallmark feature of mammalian annexins is the presence of the endonexin sequence KGXGT$\{38\}-\mathrm{D} / \mathrm{E}^{26}$ in the loops of the individual repeats where the sequence KGXGT is located in the AB loop, and the bidentate residue at bout 38 positions downstream is situated in the loop connecting helices D and E. Analysis of the primary structure of alpha-giardins reveals that this sequence is absent in most alpha-giardins; only repeat II in $\alpha 14$-giardin carries this canonical calcium binding sequence. At tertiary structure level, the endonexin sequence constitutes the type II calcium binding site situated in the AB loops of each repeat in mammalian annexins. Due to the lack of this motif in alpha-giardins, it is not surprising that novel calcium binding sites are observed in alpha-giardin crystal structures.

The level of conservation of calcium coordination between E annexins and those of other groups appears to be higher for type III sites which are present in two of three alpha-giardin structures known so far. The comparison of calcium-bound $\alpha 1$-, $\alpha 11$ - and $\alpha 14$-giardin crystal structures also reveals that these proteins explore several different calcium coordination schemes. While the slow rate of change in molecular evolution and the concomitantly high conservation of the annexin fold reflect a strong preservation of function ${ }^{27}$, the variety of calcium binding sites in alpha-giardins may represent snapshots of the evolution of canonical metal binding sites in this ubiquitous protein family. Yet all sites known so far are located on the convex surface, thus indicating that the membrane binding site is an invariant feature of annexins of all groups. 


\section{A model for glycosaminoglycan binding}

Lectin properties in form of binding to heparan sulphate have previously been reported for $\alpha 1$ giardin $^{21}$. Results from the heparin binding assay in the present study agree with this finding, but also enable more detailed insights into the mechanisms of interaction. In the absence of calcium, about 20\% binding to Heparin Sepharose is observed. These interactions are most likely mediated by small basic surface patches, which are especially prominent on the convex surface of the protein. Of particular importance in this respect may be the KK and KR motifs in repeats I (Lys55, Lys56), II (Lys119, Lys120) and IV (Lys282, Arg283). Results from the calcium titration series clearly indicate a cooperative binding behaviour with a Hill number close to 2. In the calcium-bound crystal structure of $\alpha 1$-giardin two occupied calcium sites are observed, located on the I/IV module. We therefore propose that these two protein-bound calcium ions mediate the calcium-dependent glycosaminoglycan interactions of $\alpha 1$-giardin. This notion is further supported by the basic amino acids in the immediate environment of the calcium ions, namely Lys55, Lys56 and Lys 59 around Ca300, and Lys282 and Arg283 in the direct vicinity of Ca301. Attempts to obtain crystals of $\alpha 1-$ giardin bound to heparin-derived tetrasaccharides by co-crystallisation and soaking have remained unsuccessful to date.

These findings resemble the situation found with annexin B1 from the parasite Cysticercus cellulosae which also shows cooperative calcium-dependent binding to Heparin Sepharose ${ }^{22}$. In contrast to annexin B1, however, $\alpha 1$-giardin exhibits no irreversible binding to heparin thereby revealing a distinctly different glycosaminoglycan interaction mechanism. For annexin B1 and human annexin A5, models have been put forward that propose a shift of the binding mode after the initial calcium-mediated step to a calcium-independent, irreversible attachment to glycosaminoglycans. This model, initially proposed by Seaton and colleagues ${ }^{19}$, suggests that the glycosaminoglycan-bound annexin was interacting with the membrane by its convex surface while being wrapped by the glycosaminoglycan chain extending from the membrane surface. The absence 
of this irreversible binding mode in the case of $\alpha 1$-giardin suggests that this protein does not engage a shift in interaction mechanism and solely relies on calcium mediation.

Notably, there is no deviation from the saturated binding curve at high calcium concentrations. In contrast to membrane binding, $\alpha 1$-giardin interactions with heparin are therefore not affected by dimer formation at calcium concentrations beyond $5 \mathrm{mM}$. The proposed head-to-head dimer is in agreement with this observation, as there is ample access to the presumed heparin binding site(s) on the convex surface enabling an intact dimer with a protruding heparin chain. In the absence of an experimental structure of glycosaminoglycan-bound $\alpha 1$-giardin, we cannot exclude the possibility, though, that the heparin binding sites overlap with the dimer interface. This latter scenario would render $\alpha 1$-giardin in the monomeric state at high calcium concentrations, but also be in agreement with the observed heparin binding profile.

\section{Calcium-regulated membrane binding mechanism}

$\alpha 1$-giardin binds to acidic phospholipid membranes with a clear requirement for phosphatidylserine as well as calcium. Despite the presence of exposed basic residues on the convex surface of the protein, $\alpha 1$-giardin does not associate with membranes in the absence of calcium, as observed for example with plant annexins ${ }^{28}$, presumably because it lacks hydrophobic surface residues that aid in anchoring the protein in the membrane.

Results from size exclusion chromatography and small-angle X-ray scattering in this study show that $\alpha 1$-giardin exists as a dimeric species in solution in the absence of calcium. The results further demonstrate that in the presence of $<5 \mathrm{mM}$ calcium the protein monomerises and interacts with acidic phospholipid membranes, displaying a typical calcium-dependent membrane binding behaviour known from other annexins. At calcium concentrations higher than $5 \mathrm{mM}$, increasing amounts of $\alpha 1$-giardin disengage from the membrane surface, presumably because a dimeric species is formed that renders the membrane binding surface inaccessible for interactions with 
membrane-embedded phospholipids. The most likely species explaining this behaviour comprises a head-to-head dimer of $\alpha 1$-giardin which may be stabilised by additional calcium ions bound by residues of the convex surfaces of either monomer (see Figure 6).

Interestingly, within the life cycle of Giardia, the cyst is digested by enzymes in the stomach where the calcium concentration is between 5 and $50 \mathrm{mM}^{29}$. In this milieu of high calcium concentration, $\alpha 1$-giardin, which provides structural integrity in the cyst as well as the trophozoite stages, is triggered to disengage from the plasma membrane by high environmental calcium concentrations experienced by the excyzoite. The less rigid microribbon structures can then undergo the large structural transformations required to form the trophozoite. In the duodenum, calcium is absorbed by the host mucosa, thereby limiting the calcium pool available to the parasite. In this situation it is hypothesised $\alpha 1$-giardin could resume its reinforcing structural role and return to the plasma membrane-bound form. The suggested mechanism and the immunodominance of $\alpha 1$-giardin are further in agreement with a proposed role of the protein ensuring attachment of the excyzoite to host epithelium while the ventral adhesive disk is being assembled. Future studies will need to address the mechanistic details of $\alpha 1$-giardin secretion into the local environment as well as the impact of environmental factors such as $\mathrm{pH}$ on binding and localisation of the parasite.

\section{Conclusions}

Despite the recognised importance of the ventral disk for attachment of Giardia trophozoites to host epithelium, the detailed mechanisms of attachment remain unclear to date. A hydrodynamic model whereby flagellar movements would provide a force maintaining the suction-based attachment of the disk has recently been discarded ${ }^{30}$. Cytoskeletal components with regulated interaction properties are therefore of high interest for understanding Giardia establishment. 
A direct role of $\alpha 1$-giardin in binding to host glycosaminoglycan would also provide a mechanism of attachment of the excyzoite in the absence of a functional ventral adhesive disk during the early stages of establishment. Such a mechanism would be in agreement with the high level of anti- $\alpha 1$ giardin antibodies early during a Giardia infection ${ }^{31}$.

The present study provides structural and mechanistic insights into the elaborate calcium-regulated and -mediated mechanisms by $\alpha 1$-giardin. Given the crucial importance of this protein for the structural integrity and thus survival of the parasite in the host intestine, we believe that $\alpha 1$-giardin is a worthwhile target for therapeutic interference. If the membrane or glycosaminoglycan binding activity of the protein can be suppressed after its disassembly from the plasma membrane during excystation, the transformation process to the trophozoite will be stalled and compromise the integrity of the parasite whose remains can be cleared by the host immune system.

\section{Materials and Methods}

\section{Preparation of recombinant $\alpha 1$-giardin}

The cDNA of $\alpha 1$-giardin was subcloned into pRSET_6c ${ }^{32}$, and transformed into competent E. coli BL21(DE3) cells. Expression of $\alpha 1$-giardin followed an in-house adaptation of the auto-induction protocol described by Studier ${ }^{33}$. A total of $8 \mathrm{~L}$ of $\mathrm{LB}^{+}$auto- induction medium $\left(50 \mathrm{mg} \mathrm{L}^{-1}\right.$ ampicillin) were inoculated with an overnight culture of $1 \mathrm{~L}$. The cells were grown at $37^{\circ} \mathrm{C}$ for 4 hrs, and then incubation was continued another 48 hrs at $30^{\circ} \mathrm{C}$. After harvest, the cells were resuspended (100 mM NaCl, 1 mM EDTA, 20 mM TRIS (pH 8), 0.1\% Triton X-100, 1 mM PMSF, $5 \mathrm{mM}$ benzamidinium chloride), and lysed by multiple freeze-thaw cycles and subsequent sonication. The resulting suspension was cleared by ultracentrifugation (100000 $\left.\mathrm{g}, 30 \mathrm{~min}, 4^{\circ} \mathrm{C}\right)$. Purification of soluble $\alpha 1$-giardin was achieved by calcium-dependent adsorption/desorption 
to/from liposomes. Liposomes were prepared from PS-rich soy lipid (Lipid Products, Surrey, U.K.) and the supernatant from the ultracentrifugation step was incubated with the liposome suspension in the presence of $5 \mathrm{mM} \mathrm{CaCl}_{2}$. The liposomes were pelleted by ultracentrifugation $(100000 \mathrm{~g}, 30$ min, $4^{\circ} \mathrm{C}$ ), washed with $5 \mathrm{mM} \mathrm{CaCl}$ and the bound protein subsequently desorbed with $5 \mathrm{mM}$ EDTA and CHELEX. All buffers contained $100 \mathrm{mM} \mathrm{NaCl}, 20 \mathrm{mM}$ TRIS (pH 8). As a final purification step, anion exchange chromatography was conducted using Q-Sepharose resin, and an elution gradient of 0-1 M NaCl, $20 \mathrm{mM}$ HEPES (pH 8). Protein quality was monitored throughout all purification procedures using denaturing SDS-PAGE ${ }^{34}$.

\section{Synthesis of $\left[\mathrm{Ta}_{6} \mathrm{Br}_{12}\right] \mathrm{Br}_{2}$}

Synthesis of the tantalum bromide cluster followed the procedure by Koknat and colleagues ${ }^{35}$.

\section{Crystallisation}

All crystallisation experiments employed the vapour diffusion method and hanging drops at $16^{\circ} \mathrm{C}$. Initial screening of our large in-house factorial collection with more than 1000 pre-formulated conditions indicated a variety of crystallisation conditions for $\alpha 1$-giardin around $20 \%-30 \%$ PEG 2000-6000 at $\mathrm{pH}$ values 6-9. A crystal diffracting up to $1.9 \AA$ (dataset A1G02) was grown at $0.1 \mathrm{M}$ $\mathrm{MgCl}_{2}, 30 \%$ PEG2000, $20 \mathrm{mM} \mathrm{KH} \mathrm{PO}_{4}(\mathrm{pH}=8)$. A crystal derivatised with the tantalum bromide cluster (soaking in saturated $\left[\mathrm{Ta}_{6} \mathrm{Br}_{12}\right] \mathrm{Br}_{2}$ for 3 days; dataset $\mathrm{A} 1 \mathrm{G} 44$ ) was obtained from 20\% PEG 6000, 0.1 M TRIS (pH=9). Calcium-bound $\alpha 1$-giardin (dataset A1G46) was crystallised from 15 mM CaCl $2,20 \%$ PEG 6000, 0.1 M MES (pH=6) using micro-seeding. Crystals typically appeared after two weeks and had the shape of thin plates of about $0.5 \mathrm{~mm}$ length $(\mathrm{pH}=6)$ and short tetragonal prisms of approximate dimensions $01 \times 0.1 \times 0.2 \mathrm{~mm}^{3}(\mathrm{pH}=7-8)$. 


\section{Data collection, structure solution and refinement}

Crystals were prepared for X-ray diffraction by brief immersion in mother liquor containing 25\% glycerol and flash freezing in liquid nitrogen. X-ray diffraction data were collected at the in- house diffractometer (Rigaku MicroMax007-HF; R-Axis IV++ detector; Rigaku X-stream cryo equipment), and at synchrotron beam lines at SRS Daresbury (UK) and the Australian Synchrotron under cryogenic conditions ( $\mathrm{T}=100 \mathrm{~K}$ ). Datasets were indexed with Mosflm $^{36}$, and scaling, truncation and analysis was performed with programs from the CCP4 suite ${ }^{37}$.

Attempts to solve the crystal structure by molecular replacement with a library of 155 models derived from 17 annexin and $\alpha$-giardin structures were unsuccessful. Heavy atom derivatisation proved difficult, but a crystal soaked with $\left[\mathrm{Ta}_{6} \mathrm{Br}_{12}\right] \mathrm{Br}_{2}$ yielded a reasonable anomalous signal (RCR 4.1 in A1G44_PK). Using three MAD datasets (A1G44), three pseudo heavy atom sites were detected by SHELX ${ }^{38}$ and initial phases could be derived using SHARP ${ }^{39}$. For phasing statistics, see Supplementary Table S3. The electron density allowed manual placement of twenty $\alpha$-helices using $\mathrm{O}^{40}$ which were subsequently refined by computational rigid body refinement with Phenix ${ }^{41}$. With poor electron density in many areas, no further manual model building was possible, and we thus employed a hybrid modelling-replacement approach provided by ROSETTA and Phenix ${ }^{42}$. The resulting model was used to phase the high resolution dataset A1G2 where the final model building was conducted. The calcium-bound structure of $\alpha 1$-giardin was solved by molecular replacement. The geometry of the final models was scrutinised with PROCHECK ${ }^{43}$. Data collection and refinement statistics are summarised in Table 1 . Coordinates and structure factors have been deposited with the PDB (PDB ID: 4EVF, PDB ID: 4EVH).

\section{Thermal stability}

Thermal denaturation of $\alpha 1$-giardin in the absence and presence of calcium was monitored by circular dichroism (CD) using a Jasco J-715 spectropolarimeter equipped with a Peltier element. 
Protein samples with final concentration of $1.8 \mu \mathrm{M}$ were prepared in $50 \mathrm{mM} \mathrm{NaCl}, 5 \mathrm{mM}$ TRIS (pH 8) in the presence and absence of $5 \mathrm{mM} \mathrm{CaCl}_{2}$. The $\mathrm{CD}$ signal at $222 \mathrm{~nm}$ was monitored, while heating from $20^{\circ} \mathrm{C}$ to $80^{\circ} \mathrm{C}$ at $0.5 \mathrm{~K}$ intervals spaced by 1 min equilibration time. Changes in the CD at $222 \mathrm{~nm}$ were used to construct an unfolding curve. Three independent experiments were conducted for each calcium condition. Data were plotted and smoothed using the software SDAR ${ }^{44}$.

\section{Calcium-dependent aggregation/precipitation assay}

Calcium-induced precipitation of $\alpha 1$-giardin was assessed as a control for the heparin binding and liposome copelleting assays. $10 \mu \mathrm{L}$ of $2 \mathrm{mg} \mathrm{mL}^{-1}$ protein in standard buffer (100 mM NaCl, $20 \mathrm{mM}$ HEPES, pH 7.5) or liposome buffer was added to $390 \mu \mathrm{L}$ of standard buffer containing different concentrations of calcium $(0-10 \mathrm{mM})$ and incubated for $10 \mathrm{~min}$ at room temperature. After centrifugation (30 $\min , 16000 \mathrm{~g}, 4^{\circ} \mathrm{C}$ ), the supernatant were carefully removed. The tubes were washed with $20 \mu \mathrm{L}$ of $10 \%$ SDS and the samples subjected to SDS-PAGE. Gels were stained with Coomassie Brilliant Blue and analysed densitometrically using the program ImageJ ${ }^{45}$.

\section{Liposome co-pelleting assay}

Phospholipid vesicles were prepared from 1,2-dioleoyl-sn-glycero-3-phosphoserine and 1,2dioleoyl-sn-glycero-3-phosphocholine (Avanti Polar Lipids, Alabaster, AL, USA) according to the protocol of Reeves \& Dowben ${ }^{46}$. The vesicles were converted into LUVs using five freeze-thaw cycles and subsequent extrusion (11 times) through $0.1 \mu \mathrm{m}$ filter membranes using an extruder (Avanti Polar Lipids) at $37^{\circ} \mathrm{C}$. To assess the annexin-membrane binding behaviour, a copelleting assay was conducted ${ }^{47}$. A total of $0.2 \mu$ mol phospholipids was used for each individual sample (500 $\mu \mathrm{L})$, composed of $0.5 \mathrm{nmol}$ protein in liposome buffer and varying amounts of calcium (0-20 mM). As a control, a sample of $0.1 \mathrm{nmol}$ protein in $100 \mu \mathrm{L}$ of $10 \%$ SDS was prepared at this stage. All samples were centrifuged (45 $\mathrm{min}, 16000 \mathrm{~g}, 4^{\circ} \mathrm{C}$ ), the pellets resuspended with $50 \mu \mathrm{L}$ of $10 \%$ SDS 
and subjected to SDS-PAGE. Gels were stained with Coomassie Brilliant Blue and analysed densitometrically using the program Image ${ }^{45}$. The assay was conducted in triplicate for each calcium concentration.

\section{Heparin binding assay}

Calcium-dependent binding of annexins to heparin was investigated using a centrifugation assay with Heparin Sepharose resin (Amersham Pharmacia Biotech, Piscataway, NJ, USA) following a previously published procedure ${ }^{22} .1 \mathrm{~mL}$ of well-suspended resin was equilibrated in centrifuge tubes by washing three times with buffer containing $100 \mathrm{mM} \mathrm{NaCl}, 20 \mathrm{mM}$ TRIS (pH 8.0). All centrifugation steps were carried out at $300 \mathrm{rpm}$ for $3 \mathrm{~min}$ at $4^{\circ} \mathrm{C}$. Individual aliquots of resin were resuspended in protein buffer (final volume $390 \mu \mathrm{L}$ each) with varying concentrations of $\mathrm{CaCl}_{2}(0$, 0.25, $0.50,1.0,2.0,5.0$, and $10 \mathrm{mM}$ ). Finally, $10 \mu \mathrm{L}$ of protein (ca. $5 \mathrm{mg} \mathrm{mL}^{-1}$ ) was added to each aliquot. After 10 min incubation at room temperature, the samples were subjected to centrifugation. The pellets were then washed with $400 \mu \mathrm{L}$ buffer containing the appropriate calcium concentration. The protein was eluted with $400 \mu \mathrm{L}$ of protein buffer containing $30 \mathrm{mM}$ EDTA. After centrifugation, the supernatant was analysed by SDS-PAGE. The amount of reversibly bound annexin was determined by densitometric analysis of Coomassie Brilliant Blue-stained SDS-PAGE of the final elution fraction using the ImageJ program ${ }^{45}$. All calcium conditions were assessed in triplicate, and the data were fitted using $\mathrm{SDAR}^{44}$.

\section{Size exclusion chromatography}

Size exclusion chromatography was carried out using a Superose 12 column (GE Healthcare) on a BioLogic HPLC system (BioRad), previously calibrated with protein standards. All experiments were performed at a flow rate of $0.5 \mathrm{~mL} \mathrm{~min}^{-1}$, and isocratic elution with a buffer containing 100 $\mathrm{mM} \mathrm{NaCl}, 20 \mathrm{mM}$ HEPES (pH 7.5), in the absence or presence of $10 \mathrm{mM} \mathrm{CaCl}_{2}$. After 
equilibrating the column, $\alpha 1$-giardin at a concentration of $5 \mathrm{mg} \mathrm{mL}^{-1}$ was loaded and its elution monitored by UV absorbance at $280 \mathrm{~nm}$. The chromatograms were analysed using the program $\mathrm{SDAR}^{44}$ to determine the position of and area under the elution peaks.

\section{Small-angle X-ray scattering and molecular modelling}

Small-angle X-ray scattering data of $\alpha 1$-giardin in the absence and presence of various calcium concentrations were acquired at the SAXS/WAXS beamline of the Australian Synchrotron. The sample-to-detector distance was $1600 \mathrm{~mm}$, which covered a momentum transfer range of $0.006<q$ $<0.313 \AA^{-1}$, where $q=(4 \pi \sin \theta) / \lambda, 2 \theta$ is the scattering angle, and $\lambda$ is the radiation wavelength (1.033 $\AA$ ). $50 \mu \mathrm{l}$ of each sample was flowing through the beam in a $1.5 \mathrm{~mm}$ quartz capillary at room temperature, and frames with 2 sec exposure were acquired.

Data reduction and averaging of 5 frames was done with SAXS15ID software ${ }^{48}$. All data were corrected for background scattering from $100 \mathrm{mM} \mathrm{NaCl}, 20 \mathrm{mM}$ HEPES (pH 8.0), and the scattering intensities were transformed to absolute units using the scattering of water as standard. Concentration series for all samples were recorded at $\rho^{*}, \rho^{* / 2}$ and $\rho^{* / 4}$, with $\rho^{*}$ ranging from 8 to $15 \mathrm{mg} \mathrm{mL}^{-1}$.

The radius of gyration $R_{g}$, intensity of forward scattering $I(0)$ were calculated using $\mathrm{GNOM}^{49}$. The experimental molecular mass was calculated from the intensity of forward scattering $I(0)$ using the formula:

$\mathrm{M}=I(0) * N_{A} /\left[\left(\rho^{*} / 1000\right) * \Delta \rho^{2}\right]$, where $\Delta \rho=2.0 * 10^{10} \mathrm{~cm} \mathrm{~g}^{-1}$ is the scattering contrast and $\mathrm{N}_{\mathrm{A}}=$ $6.022 * 10^{23} \mathrm{~mol}^{-1}$ is Avogadro's constant.

Data quality was assessed by inspection of the concentration dependence of the scattering data and linearity of the Guinier region. For all datasets, the Guinier plot $\left(\ln I(q)\right.$ vs. $\left.q^{2}\right)$ was linear, and $I(0)$ 
values normalised by concentration showed no systematic trend, with exception of the samples measured in the presence of $10 \mathrm{mM} \mathrm{CaCl}_{2}$ (see Supplementary Table S3).

\section{References}

1. Geurden, T., Vercruysse, J. \& Claerebout, E. (2006). Field testing of a fenbendazole treatment combined with hygienic and management measures against a natural Giardia infection in calves. Vet. Parasitol. 142, 367-371.

2. Savioli, L., Smith, H. \& Thompson, A. (2006). Giardia and Cryptosporidium join the 'Neglected Diseases Initiative'. Trends Parasitol. 22, 203-208.

3. Lalle, M. (2010). Giardiasis in the post genomic era: treatment, drug resistance and novel therapeutic perspectives. Infect Disord Drug Targets 10, 283-294.

4. Elmendorf, H.G., Dawson, S.C. \& McCaffery, J.M. (2003). The cytoskeleton of Giardia lamblia. Int. J. Parasitol. 33, 3-28.

5. Fiedler, K. \& Simons, K. (1995). Annexin homologues in Giardia lamblia. Trends Biochem. Sci. 20, 177-178.

6. Bauer, B., Engelbrecht, S., Bakker-Grunwald, T. \& Scholze, H. (1999). Functional identification of alpha 1-giardin as an annexin of Giardia lamblia. FEMS Microbiol. Lett 173, 147153.

7. Weber, K., Geisler, N., Plessmann, U., Bremerich, A., Lechtreck, K.F. \& Melkonian, M. (1993). SF-assemblin, the structural protein of the 2-nm filaments from striated microtubule associated fibers of algal flagellar roots, forms a segmented coiled coil. J Cell Biol 121, 837-845.

8. Nohria, A., Alonso, R.A. \& Peattie, D.A. (1992). Identification and characterization of gamma-giardin and the gamma-giardin gene from Giardia lamblia. Mol Biochem Parasitol 56, 2737.

9. Hofmann, A., Osman, A., Leow, C.Y., Driguez, P., McManus, D.P. \& Jones, M.K. (2010). Parasite annexins - new molecules with potential for drug and vaccine development. BioEssays 32, 967-976.

10. Morrison, H.G., McArthur, A.G., Gillin, F.D., Aley, S.B., Adam, R.D., Olsen, G.J., Best, A.A., Cande, W.Z., Chen, F., Cipriano, M.J., Davids, B.J., Dawson, S.C., Elmendorf, H.G., Hehl, A.B., Holder, M.E., Huse, S.M., Kim, U.U., Lasek-Nesselquist, E., Manning, G., Nigam, A., Nixon, J.E.J., Palm, D., Passamaneck, N.E., Prabhu, A., Reich, C.I., Reiner, D.S., Samuelson, J., Svard, S.G. \& Sogin, M.L. (2007). Genomic minimalism in the early diverging intestinal parasite Giardia lamblia. Science 317, 1921-1926.

11. Peattie, D.A., Alonso, R.A., Hein, A. \& Caulfield, J.P. (1989). Ultrastructural localization of giardins to the edges of disk microribbons of Giardia lamblia and the nucleotide and deduced protein sequence of alpha giardin. J. Cell Biol. 109, 2323-2335.

12. Weiland, M., McArthur, A., Morrison, H., Sogin, M. \& Svärd, S. (2005). Annexin-like alpha giardins: a new cytoskeletal gene family in Giardia lamblia. Int. J. Parasitol. 35, 617-626.

13. Palm, D., Weiland, M., McArthur, A.G., Winiecka-Krusnell, J., Cipriano, M.J., Birkeland, S.R., Pacocha, S.E., Davids, B., Gillin, F., Linder, E. \& Svärd, S. (2005). Developmental changes in the adhesive disk during Giardia differentiation. Mol Biochem Parasitol 141, 199-207.

14. Roxström-Lindquist, K., Palm, D., Reiner, D., Ringqvist, E. \& Svärd, S.G. (2006). Giardia immunity--an update. Trends Parasitol 22, 26-31.

15. Skehel, J.J. \& Wiley, D.C. (2000). Receptor binding and membrane fusion in virus entry: the influenza hemagglutinin. Annu Rev Biochem 69, 531-569. 
16. Reeder, J.C., Cowman, A.F., Davern, K.M., Beeson, J.G., Thompson, J.K., Rogerson, S.J. \& Brown, G.V. (1999). The adhesion of Plasmodium falciparum-infected erythrocytes to chondroitin sulfate A is mediated by P. falciparum erythrocyte membrane protein 1. Proc Natl Acad Sci U S A 96, 5198-5202.

17. Esko, J.D.. (1999). Proteins that recognise glycans. Microbial carbohydrate-binding proteins. In Essentials of Glycobiology. Varki, A., Cummings, R., Esko, J.D., Freeze, H., Hart, G. \& Marth, J. (Eds.). Cold Spring Harbor Laboratory Press, Cold Spring Harbor, New York. .

18. Tsujii-Hayashi, Y., Kitahara, M., Yamagaki, T., Kojima-Aikawa, K. \& Matsumoto, I. (2002). A potential endogenous ligand of annexin IV in the exocrine pancreas. Carbohydrate structure of GP-2, a glycosylphosphatidylinositol-anchored glycoprotein of zymogen granule membranes. $J$ Biol Chem 277, 47493-47499.

19. Capila, I., Hernaiz, M., Mo, Y., Mealy, T., Campos, B., Dedman, J., Linhardt, R. \& Seaton, B. (2001). Annexin V-heparin oligosaccharide complex suggests heparan sulfate-mediated assembly on cell surface. Structure 9, 57-64.

20. Takagi, H., Asano, Y., Yamakawa, N., Matsumoto, I. \& Kimata, K. (2002). Annexin 6 is a putative cell surface receptor for chondroitin sulfate chains. J Cell Sci 115, 3309-3318.

21. Weiland, M., Palm, J., Griffiths, W., McCaffery, J. \& Svärd, S. (2003). Characterisation of alpha-1 giardin: an immunodominant Giardia lamblia annexin with glycosaminoglycan-binding activity. Int. J. Parasitol. 33, 1341-1351.

22. Winter, A., Yusof, A.M., Gao, E., Yan, H.L. \& Hofmann, A. (2006). Biochemical characterization of annexin B1 from Cysticercus cellulosae. FEBS J. 273, 3238-3247.

23. Pathuri, P., Nguyen, E.T., Svard, S.G. \& Luecke, H. (2007). Apo and Calcium-bound Crystal Structures of Alpha-11 Giardin, an Unusual Annexin from Giardia lamblia. J. Mol. Biol. 368, 493508.

24. Pathuri, P., Nguyen, E.T., Ozorowski, G., Svärd, S.G. \& Luecke, H. (2009). Apo and calcium-bound crystal structures of cytoskeletal protein alpha-14 giardin (annexin E1) from the intestinal protozoan parasite Giardia lamblia. J. Mol. Biol. 385, 1098-1112.

25. Jenikova, G., Hruz, P., Andersson, M.K., Tejman-Yarden, N., Ferreira, P.C.D., Andersen, Y.S., Davids, B.J., Gillin, F.D., Svärd, S.G., Curtiss, R.3. \& Eckmann, L. (2011). A1-giardin based live heterologous vaccine protects against Giardia lamblia infection in a murine model. Vaccine 29, 9529-9537.

26. Geisow, M., Fritsche, U., Hexham, J., Dash, B. \& Johnson, T. (1986). A consensus amino acid sequence repeat in Torpedo and mammalian calcium-dependent membrane binding proteins. Nature 320, 636-638.

27. Benz, J. \& Hofmann, A. (1997). Annexins - From structure to function. Biol. Chem. 378, 177-183.

28. Dabitz, N., Hu, N.J., Yusof, A.M., Tranter, N., Winter, A., Daley, M., Zschörnig, O., Brisson, A. \& Hofmann, A. (2005). Structural determinants for plant annexin-membrane interactions. Biochemistry 44, 16292-16300.

29. Reiner, D., Hetsko, M., Meszaros, J., Sun, C., Morrison, H., Brunton, L. \& Gillin, F. (2003). Calcium signaling in excystation of the early diverging eukaryote, Giardia lamblia. J. Biol. Chem 278, 2533-2540.

30. House, S.A., Richter, D.J., Pham, J.K. \& Dawson, S.C. (2011). Giardia flagellar motility is not directly required to maintain attachment to surfaces. PLoS Pathog 7, e1002167.

31. Palm, J., Weiland, M., Griffiths, W., Ljungström, I. \& Svärd, S. (2003). Immunoreactive proteins during acute human giardiasis. J. Infect. Dis. 187, 1849-1859.

32. Schoepfer, R. (1993). The pRSET family of T7 promoter expression vectors for Escherichia coli. Gene 124, 83-85.

33. Studier, F. (2005). Protein production by auto-induction in high density shaking cultures. Protein Expr. Purif. 41, 207-234. 
34. Ahn, T., Yim, S., Choi, H. \& Yun, C. (2001). Polyacrylamide gel electrophoresis without a stacking gel: use of amino acids as electrolytes. Anal. Biochem 291, 300-303.

35. Koknat, F., Parsons, J. \& Vongvusharintra, A. (1974). Metal Cluster Halide Complexes. I. Efficient Synthesis of Hydrated Hexanuclear Niobium and Tantalum Cluster Halides M6X14*8H2O. Inorg. Chem. 13, 1699-1702.

36. Leslie, A. (1992). Recent changes to the MOSFLM package for processing film and image plate data. Joint CCP4 + ESF-EAMCB Newsletter on Protein Crystallography 26, .

37. Collaborative Computational Project Number 4 (1994). The CCP4 suite: programs for protein crystallography. Acta Crystallogr. D50, 760-763.

38. Schneider, T. \& Sheldrick, G. (2002). Substructure solution with SHELXD. Acta Crystallogr. D 58, 1772-1779.

39. Vonrhein, C., Blanc, E., Roversi, P. \& Bricogne, G. (2007). Automated structure solution with autoSHARP. Methods Mol. Biol. 364, 215-230.

40. Jones, T.A., Zou, J.Y., Cowan, S. \& Kjeldgaard, M. (1991). Improved methods for building protein models in electron density maps and location of errors in these models. Acta Crystallogr. A 47, 110-119.

41. Adams, P.D., Afonine, P.V., Bunkóczi, G., Chen, V.B., Davis. I. W., Echols, N., Headd, J.J., Hung, L., Kapral, G.J., Grosse-Kunstleve, R.W., McCoy, A.J., Moriarty, N.W., Oeffner, R., Read, R.J., Richardson, D.C., Richardson, J.S., Terwilliger, T.C. \& Zwart, P.H. (2010). PHENIX: a comprehensive Python-based system for macromolecular structure solution. Acta Crystallogr. D66, 213-221.

42. Terwilliger, T.C., Dimaio, F., Read, R.J., Baker, D., Bunkóczi, G., Adams, P.D., GrosseKunstleve, R.W., Afonine, P.V. \& Echols, N. (2012). phenix.mr_rosetta: molecular replacement and model rebuilding with Phenix and Rosetta. J Struct Funct Genomics in press, .

43. Laskowski, R., MacArthur, M., Moss, D. \& Thornton, J. (1993). PROCHECK: A program to check the stereochemical quality of protein structures. J. Appl. Cryst. 26, 283-291.

44. Hofmann, A. \& Wlodawer, A. (2002). PCSB - a program collection for structural biology and biophysical chemistry. Bioinformatics 18, 209-210.

45. Rasband, W. (2005). ImageJ, version 1.30. National Institutes of Health, Bethesda, MD, http://rsb.info.nih.gov/ij/, .

46. Reeves, J. \& Dowben, R. (1969). Formation and properties of thin-walled phospholipid vesicles. J. Cell. Physiol 73, 49-60.

47. Hofmann, A. \& Huber, R. (2003). Liposomes in assessment of annexin-membrane interactions. Methods Enzymol. 372, 186-216.

48. Cookson, D.J. (2007). Saxs15ID - Software for acquiring, processing and viewing SAXS/WAXS image data at ChemMatCARS . http://cars9.uchicago.edu/chemmat/pages/swsoftware.html , .

49. Semenyuk, A. \& Svergun, D. (1991). GNOM: a program package for small-angle scattering data processing. J. Appl. Crystallogr. 24, 537-540.

50. Brünger, A. (1992). Free R value: a novel statistical quantity for assessing the accuracy of crystal structures. Nature 355, 472-475.

51. Kleywegt, G.J. (1996). MOLEMAN2. http://xray.bmc.uu.se/usf/ , .

52. Ortega, A., Amoros, D. \& de la Torre, J.G. (2011). Prediction of hydrodynamic and other solution properties of rigid proteins from atomic- and residue-level models. Biophys J 101, 892898.

53. DeLano, W. (2002). The PyMOL Molecular Graphics System. http://www.pymol.org , . 


\section{Acknowledgements}

This study was supported in parts by the Rebecca Cooper Foundation (AH), James N Kirby Foundation (AH), and the National Health and Medical Research Council (AH, MJ). CKW is supported by an National Health and Medical Research Council postdoctoral training fellowship (37677). This research was undertaken partially on the MX and SAXS/WAXS beamlines at the Australian Synchrotron, Victoria, Australia, and the Synchrotron Radiation Source in Daresbury, Warrington, UK. The help of Dr Kevin Jack (University of Queensland) with SAXS data acquisition is gratefully acknowledged. We also thank members of the Hofmann lab who have contributed to this project over the years.

\section{Additional information \\ Supplementary Information accompanies this paper.}

Competing financial interests: The authors declare no competing financial interests. 


\section{Tables}

Table 1

Data collection and refinement statistics for the crystal structures of apo- and calcium-bound $\alpha 1-$ giardin

\begin{tabular}{|c|c|c|}
\hline Data set & A1G2 & A1G46 \\
\hline Protein & Apo $\alpha 1$-giardin & Calcium-bound $\alpha 1$-giardin \\
\hline \multicolumn{3}{|l|}{ Data collection } \\
\hline X-ray source & SRS Daresbury & AS-MX2 \\
\hline Detector & & Quantum ADSC \\
\hline Wavelength $(\AA)$ & 1.4880 & 1.37764 \\
\hline Space group & $\mathrm{P} 22_{1} 2_{1}$ & $\mathrm{P} 2_{1} 2_{1} 2_{1}$ \\
\hline Cell dimensions $(\AA)$ & 51.3, 58.7, 99.3 & 51.6, 58.5, 98.8 \\
\hline Max. resolution $(\AA)$ & 1.9 & 2.6 \\
\hline Wilson B-factor $\left(\AA^{2}\right)$ & 25.7 & 44.2 \\
\hline No of unique reflections & 23924 (3386) & $9676(1360)$ \\
\hline Multiplicity & $5.1(4.8)$ & $0.998(1.00)$ \\
\hline Completeness & $0.983(0.972)$ & $5.5(5.6)$ \\
\hline $\mathrm{R}_{\text {sym }}{ }^{\mathrm{a}}$ & $0.123(0.382)$ & $0.090(0.248)$ \\
\hline \multicolumn{3}{|l|}{ Refinement } \\
\hline No of reflections in working / test set & $20902 / 1126$ & $8341 / 416$ \\
\hline No of non-H protein atoms & 2367 & 2367 \\
\hline Visible residues & $2-295$ & $2-295$ \\
\hline No of water molecules & 286 & 103 \\
\hline No of ions & 0 & $2\left(\mathrm{Ca}^{2+}\right)$ \\
\hline \multicolumn{3}{|l|}{ Average B-factor $\left(\AA^{2}\right)$} \\
\hline Protein & 25.7 & 26.4 \\
\hline Solvent & 32.0 & 22.8 \\
\hline Ions & & 41.0 \\
\hline rmsd B-factor for bonded atoms & 3.02 & 4.35 \\
\hline rmsd bond lengths ( $\AA$ ) & 0.007 & 0.009 \\
\hline rmsd bond angles $\left({ }^{\circ}\right)$ & 0.972 & 1.202 \\
\hline $\begin{array}{l}\text { Ramachandran plot (\%) } \\
\text { most favoured / additionally allowed / } \\
\text { generously allowed / disallowed }\end{array}$ & $93.7 / 5.9 / 0.4 / 0$ & $89.3 / 10.0 / 0.7 / 0$ \\
\hline R-factor ${ }^{\mathrm{b}}$ & $0.199(0.302)$ & $0.213(0.264)$ \\
\hline $\mathrm{R}_{\text {free }}{ }^{\mathrm{c}}$ & $0.262(0.382)$ & $0.304(0.368)$ \\
\hline
\end{tabular}

Values in parentheses refer to the last resolution shell.

${ }^{a} R_{\text {sym }}=\Sigma|\mathrm{I}-<\mathrm{I}>| / \Sigma \mathrm{I}$, where I is the observed intensity, and $<\mathrm{I}>$ is the average intensity obtained from multiple observations of symmetry-related reflections after rejections.

${ }^{\mathrm{b}} \mathrm{R}$-factor $=\Sigma|| \mathrm{F}_{\mathrm{o}}|-| \mathrm{F}_{\mathrm{c}}|| / \Sigma\left|\mathrm{F}_{\mathrm{o}}\right|$, where $\mathrm{F}_{\mathrm{o}}$ and $\mathrm{F}_{\mathrm{c}}$ are the observed and calculated structure factors, respectively. 
${ }^{\mathrm{c}} \mathrm{R}_{\text {free }}$ defined in ${ }^{50}$. 
Table 2

Comparison of experimental and modelled $R_{g}$ values.

\begin{tabular}{ccccl}
\hline $\begin{array}{c}\mathrm{c}\left(\mathrm{CaCl}_{2}\right) \\
\text { in } \mathrm{mM}\end{array}$ & $\begin{array}{c}\text { Experimental } \\
R_{g} \text { in } \AA^{\mathrm{a}}\end{array}$ & $\begin{array}{c}R_{g} \text { of protein } \\
\text { model in } \AA^{\mathrm{b}}\end{array}$ & $\begin{array}{c}\mathrm{R}_{\text {hydro }} \text { of protein } \\
\text { model in } \AA^{\mathrm{c}}\end{array}$ & \multicolumn{1}{c}{ Protein model } \\
\hline 0 & 36.2 & - & - & tail-to-tail or side-to-side dimer \\
0.1 & 30.5 & 20.2 & 27.3 & $\alpha 1$-giardin monomer crystal structure \\
10 & 33.3 & 29.1 & 38.3 & $\alpha 1$-giardin head-to-head dimer \\
\hline
\end{tabular}

${ }^{\text {a }}$ Determined using the software $\mathrm{GNOM}^{49}$ (see Materials and Methods for details).

${ }^{\mathrm{b}}$ Calculated using the software MOLEMAN2 ${ }^{51}$.

${ }^{\mathrm{C}}$ Hydrodynamic radius calculated using the software HYDROPRO ${ }^{52}$. 


\section{Figure 1}

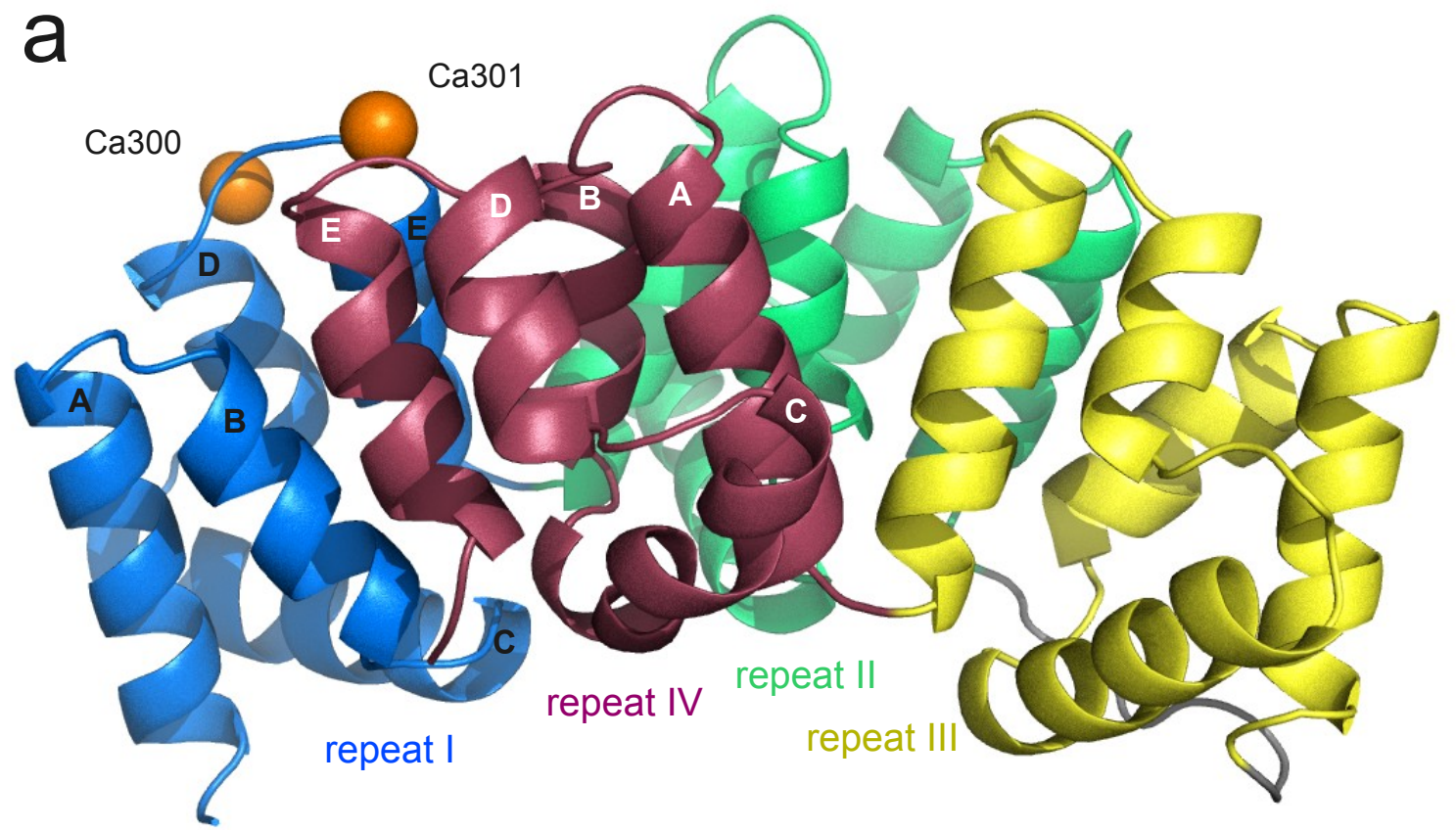

b

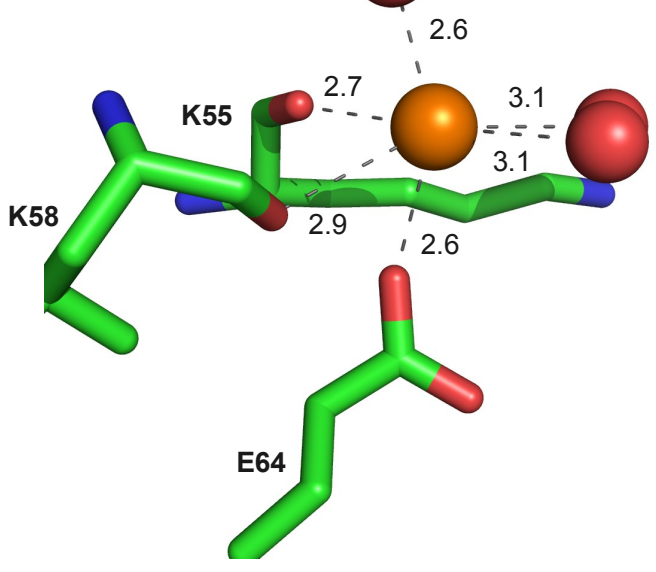

C

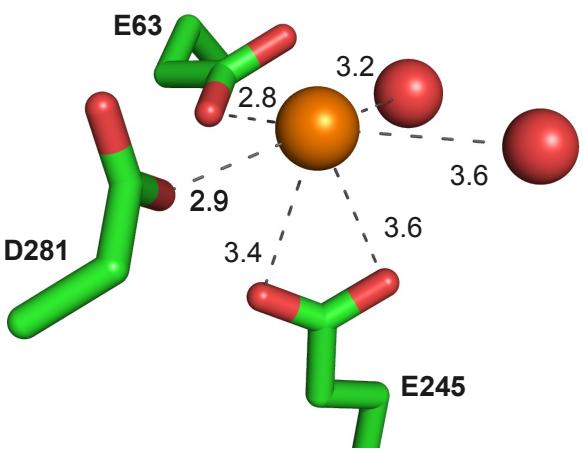

\section{Figure 1}

Crystal structure of calcium-bound $\alpha 1$-giardin. a The protein adopts the annexin fold which comprises four repeats (repeat I: blue, repeat II: green, repeat III: yellow, repeat IV: mauve). Each repeat comprises a five-helix bundle with $\mathrm{AB}$ and $\mathrm{DE}$ arranged as parallel helix-loop-helix motifs; helix $\mathrm{C}$ sits perpendicular on the side distal to the loops. The calcium ions are drawn as orange spheres. b Calcium coordination in the type III site of the first repeat. C Calcium coordination in the novel site situated between repeats I and IV. Amino acids involved in coordination are drawn as stick models, and the oxygen atom of water molecules are shown as red spheres. Coordination distances are given in $\AA$. The figure was prepared with $\mathrm{PyMol}^{53}$. 


\section{Figure 2}

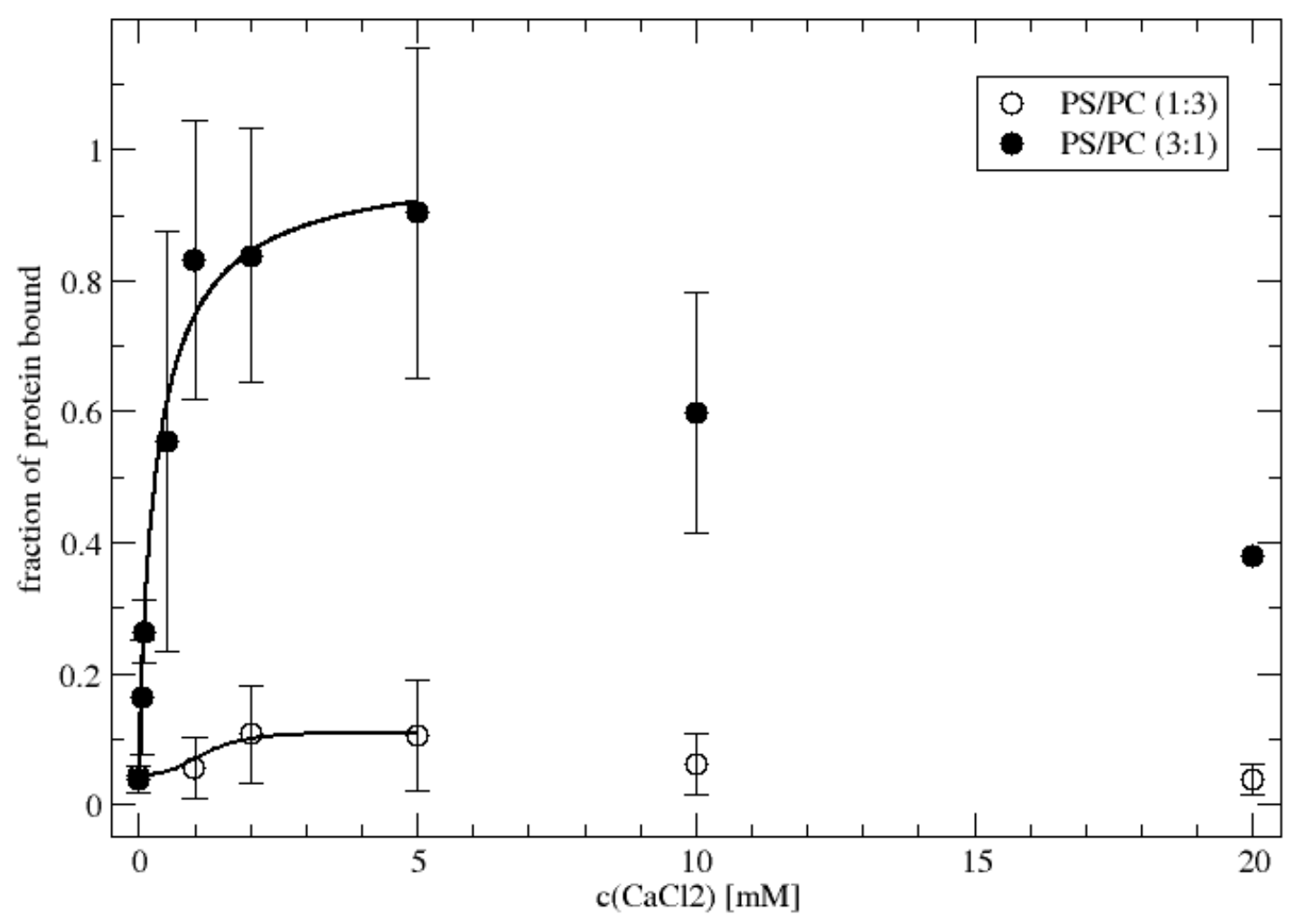

\section{Figure 2}

Calcium-dependent binding of $\alpha 1$-giardin to PS/PC (3:1; closed circles) and PS/PC (1:3; open circles) liposomes using a co-pelleting assay. The lines represent fits using the Hill equation up to $\mathrm{c}\left(\mathrm{CaCl}_{2}\right)=5 \mathrm{mM}$. Data points represent averages of three independent measurements. The fit parameters are as follows: PS/PC (3:1), $\mathrm{n}=0.95, \mathrm{k}_{\mathrm{a}}=2.9 * 10^{3} \mathrm{M}^{-1}\left(\mathrm{k}_{\mathrm{d}}=3.4 * 10^{-4} \mathrm{M}\right)$; PS/PC $(1: 3), \mathrm{n}=$ 3.2, $\mathrm{k}_{\mathrm{a}}=1.2 * 10^{3} \mathrm{M}^{-1}\left(\mathrm{k}_{\mathrm{d}}=8.3^{*} 10^{-4} \mathrm{M}\right)$. The half-maximal calcium concentrations are $\mathrm{c}_{1 / 2}=0.3 \mathrm{mM}$ for PS/PC (3:1), and $\mathrm{c}_{1 / 2}=1.1 \mathrm{mM}$ for PS/PC (1:3).

PS: phosphatidylserine; PC: phosphatidylcholine. 


\section{Figure 3}

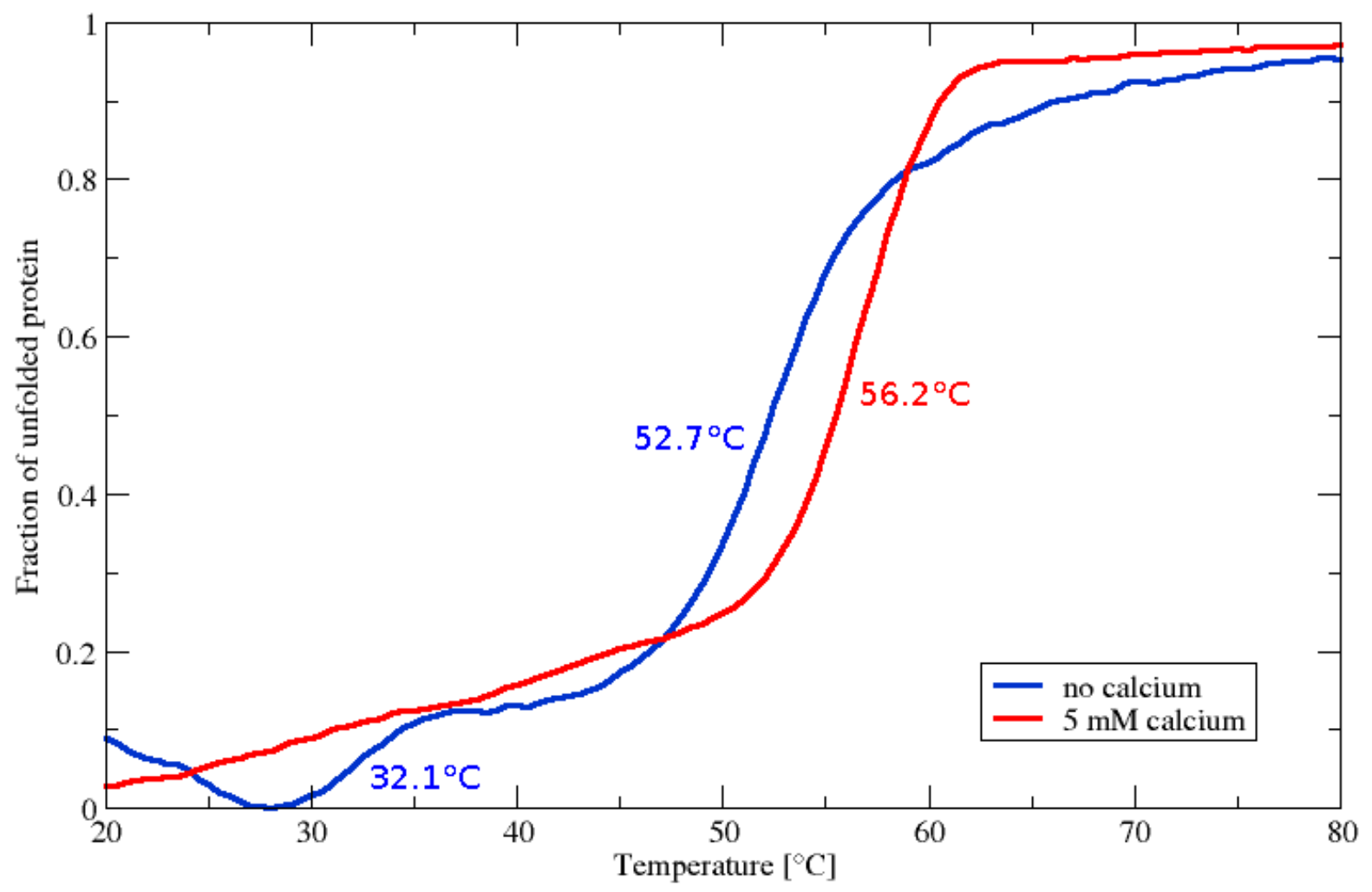

\section{Figure 3}

Thermal unfolding of $\alpha 1$-giardin in the absence (blue) and presence (red) of $5 \mathrm{mM} \mathrm{CaCl}$. The unfolding curves shown represent the smoothed averages of three independent experiments each. In the absence of calcium, two transitions are observed, one at $\mathrm{T}_{1 / 2}=32.1^{\circ} \mathrm{C}$ and one at $\mathrm{T}_{1 / 2}=52.7^{\circ} \mathrm{C}$. In the presence of $5 \mathrm{mM}$ calcium, the low temperature transition is absent, and the main transition appears at $\mathrm{T}_{1 / 2}=56.2^{\circ} \mathrm{C}$. 


\section{Figure 4}

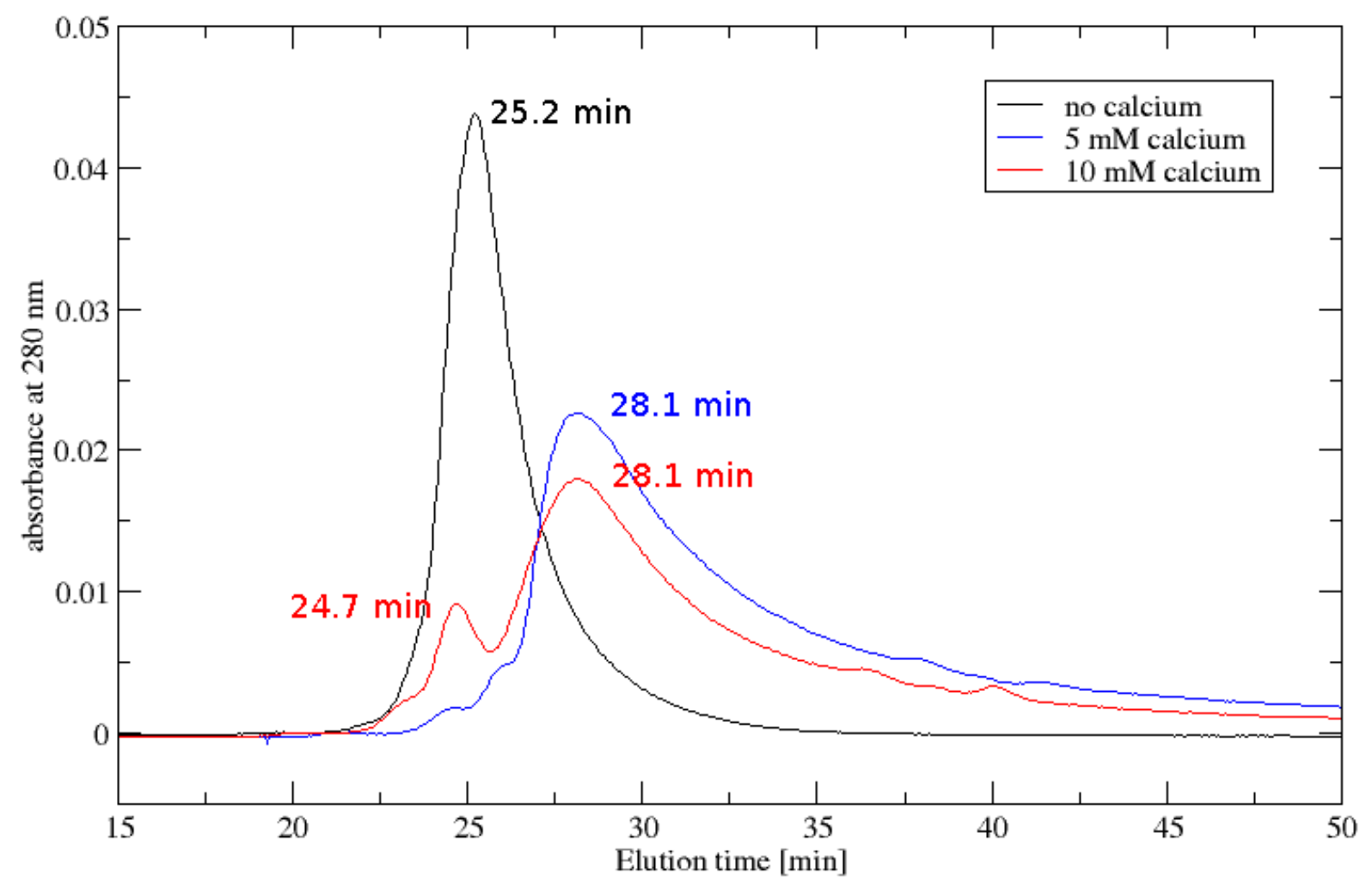

\section{Figure 4}

Size exclusion chromatography reveals calcium-dependent $\alpha 1$-giardin dimers. In the absence of calcium (black curve), a peak at 25.2 min indicates the presence of an elongated dimer, eluting at apparent lower molecular mass due to its deviation from the non-spherical shape). In the presence of $5 \mathrm{mM} \mathrm{CaCl}_{2}$ (blue curve), the main species elutes at $28.1 \mathrm{~min}$, which corresponds to monomeric $\alpha 1$-giardin. At $10 \mathrm{mM} \mathrm{CaCl}_{2}$ (red curve), two prominent species can be identified, eluting at 24.7 min (dimer) and 28.1 min (monomer). 


\section{Figure 5}

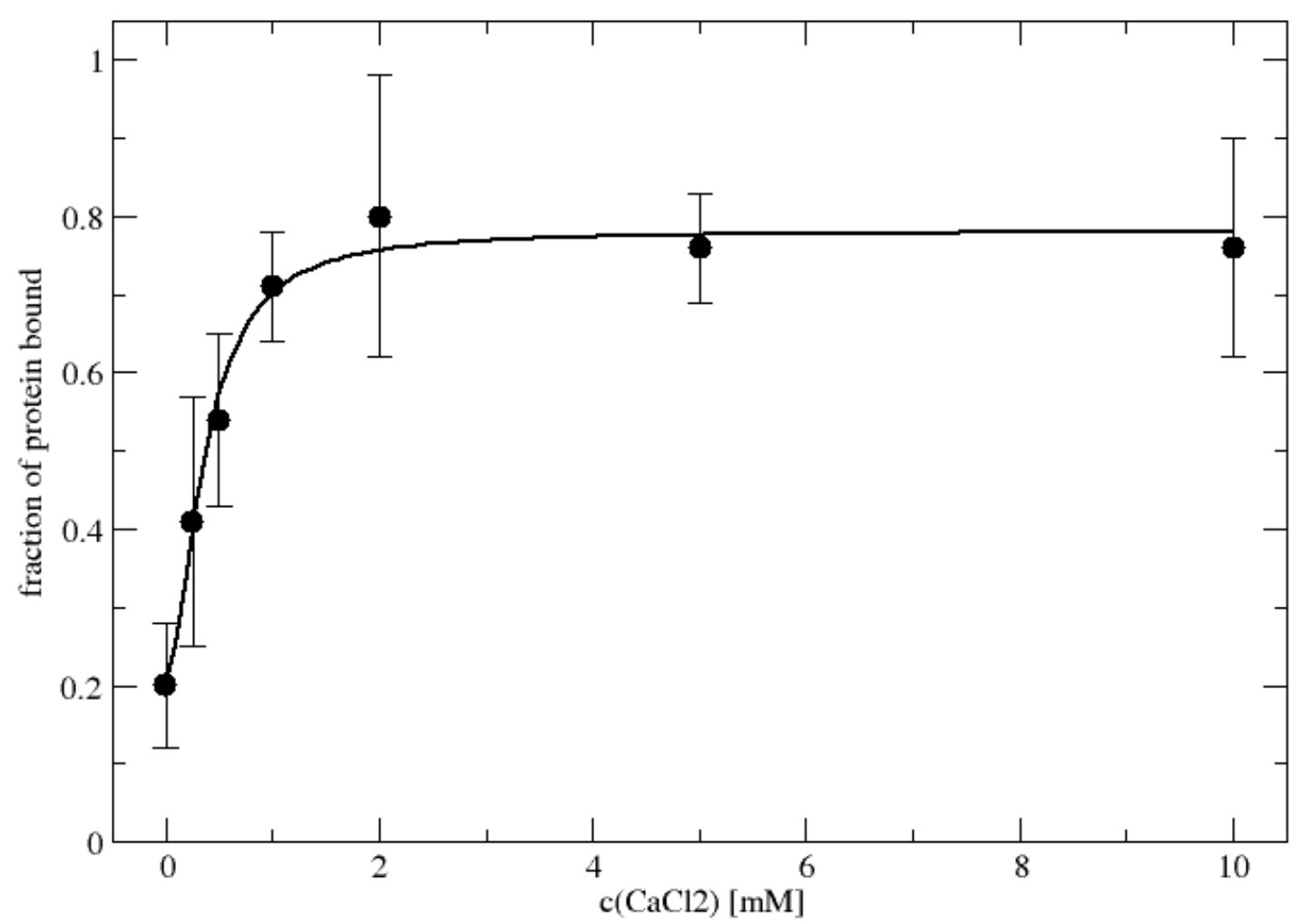

\section{Figure 5}

Calcium-dependent binding of $\alpha 1$-giardin to Heparin Sepharose. The solid line represents a fit of the experimental data using the Hill equation. The Hill number was determined to be $n=1.8$. Note the calcium-independent binding of $\alpha 1$-giardin at a fraction of $20 \%$. 


\section{Figure 6}

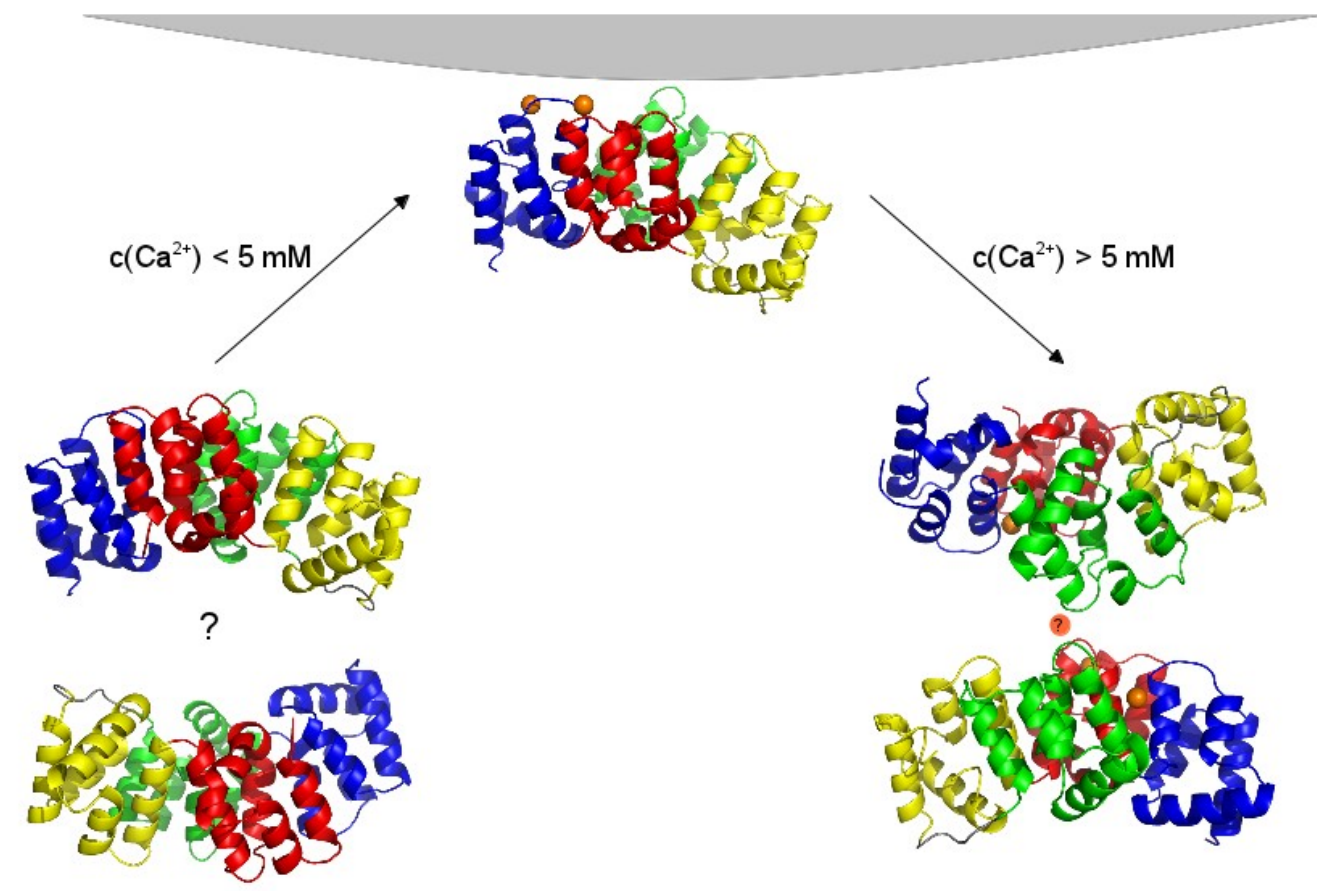

\section{Figure 6}

Results from the present study can be summarised in this model for calcium-mediated $\alpha 1$-giardin molecular mechanism. In the absence of calcium, the protein exists as a dimeric species in solution. A possible structure of this species is a tail-to-tail dimer since it renders the membrane binding sites of both molecules free for calcium binding and membrane recognition (dimer on the left). In the presence of up to $5 \mathrm{mM}$ calcium, the protein monomerises and subsequently binds to acidic phospholipid membranes and/or glycosaminoglycans. With further increasing calcium concentrations, a dimer is formed that prevents interactions with membranes, thus leading to desorption of $\alpha 1$-giardin from the membrane surface. The most likely structure for this dimer comprises a head-to-head arrangement of two $\alpha 1$-giardin molecules (dimer on the right) which renders the membrane-binding sites inaccessible but is still capable of binding to glycosaminoglycans. It is possible that this dimer involves binding of calcium ions in the dimer interface (orange sphere with question mark). 


\section{Supplementary information}

\section{Table S1}

Analysis of size exclusion chromatograms of $\alpha 1$-giardin

\begin{tabular}{cccccc}
\hline $\begin{array}{c}\mathrm{c}\left(\mathrm{CaCl}_{2}\right) \\
\text { in } \mathrm{mM}\end{array}$ & $\begin{array}{c}\text { Elution } \\
\text { time in min }\end{array}$ & $\begin{array}{c}\text { Elution } \\
\text { volume in } \mathrm{ml}\end{array}$ & $\mathrm{K}_{\mathrm{av}}$ & $\begin{array}{c}\text { Estimated } \mathrm{M} \\
\text { in } \mathrm{g} \mathrm{mol}^{-1}\end{array}$ & $\mathrm{M} /\left(33895 \mathrm{~g} \mathrm{~mol}^{-1}\right)$ \\
\hline 0 & 25.2 & 12.6 & 0.29 & 57000 & 1.7 \\
\hline 5 & 26.0 & 13.0 & 0.31 & 48000 & 1.4 \\
& 28.1 & 14.1 & 0.38 & 30000 & 0.89 \\
\hline \multirow{2}{*}{10} & 24.7 & 12.4 & 0.27 & 66000 & 1.9 \\
& 28.1 & 14.1 & 0.38 & 30000 & 0.89 \\
\hline
\end{tabular}


Table S2

Analysis of individual datasets with $\mathrm{GNOM}^{49}$ : Intensity of forward scattering, molecular mass calculation and $\mathrm{R}_{\mathrm{g}}$ values of the scattering species

\begin{tabular}{|c|c|c|c|c|c|}
\hline $\begin{array}{l}\mathrm{c}\left(\mathrm{CaCl}_{2}\right) \\
\text { in mM }\end{array}$ & $\begin{array}{c}\rho^{*}(\alpha 1 \text {-giardin }) \text { in } \\
\mathrm{mg} \mathrm{mL}^{-1}\end{array}$ & $I(0)$ in $\mathrm{cm}^{-1}$ & $\mathrm{M}$ in $\mathrm{g} \mathrm{mol}^{-1}$ & $\mathrm{M} /\left(33895 \mathrm{~g} \mathrm{~mol}^{-1}\right)$ & $\mathrm{R}_{\mathrm{g}}$ in $\AA$ \\
\hline \multirow[t]{3}{*}{0} & 2.5 & 0.09223 & 55541 & 1.6 & 36.2 \\
\hline & 5 & 0.20110 & 60551 & 1.8 & 36.2 \\
\hline & 10.3 & 0.42080 & 61506 & 1.8 & 36.2 \\
\hline \multirow[t]{3}{*}{0.1} & 2.2 & 0.07570 & 29222 & 0.9 & 30.5 \\
\hline & 4.4 & 0.16920 & 32244 & 1.0 & 30.5 \\
\hline & 8.8 & 0.33940 & 32546 & 1.0 & 30.5 \\
\hline \multirow[t]{3}{*}{10} & 2.1 & 0.04852 & 41165 & 1.0 & 33.1 \\
\hline & 4.1 & 0.10850 & 45866 & 1.2 & 33.0 \\
\hline & 8.2 & 0.28880 & 48745 & 1.6 & 33.8 \\
\hline
\end{tabular}




\section{Table S3}

Data collection and phasing statistics of the $\alpha 1$-giardin crystal structure

\begin{tabular}{|c|c|c|c|}
\hline Data set & A1G44_IN & A1G44_PK & A1G44_RM \\
\hline \multicolumn{4}{|l|}{ Crystal } \\
\hline Derivative & & {$\left[\mathrm{Ta}_{6} \mathrm{Br}_{12}\right] \mathrm{Br}_{2}$} & \\
\hline
\end{tabular}

\section{Data collection}

$\mathrm{X}$-ray source

AS-MX2

AS-MX2

AS-MX2

Detector

Wavelength $(\AA)$

Quantum ADSC

Quantum ADSC

Quantum ADSC

Space group

1.25474

1.25418

1.37756

Cell dimensions $(\AA)$

$\mathrm{P} 2{ }_{1} 2_{1} 2_{1}$

$\mathrm{P} 2{ }_{1} 2_{1} 2_{1}$

$\mathrm{P} 2{ }_{1} 2_{1} 2_{1}$

51.0, 58.8, 100.2

51.0, 58.7, 99.8

51.0, 59.0, 100.4

Resolution range $(\AA)$

5.0

5.0

3.2

52.2

50.7

55.7

Total no of measurements

18374 (2703)

18546 (2817)

56121 (8340)

1492 (206)

1482 (212)

5391 (765)

$12.3(13.1)$

12.5 (13.3)

10.4 (10.9)

$1.0(1.0)$

$1.0(1.0)$

$1.0(1.0)$

$0.066(0.105)$

$0.056(0.070)$

$0.067(0.115)$

\section{Phasing (Sharp)}

No of sites

Phasing power ${ }^{\mathrm{b}}$

isomorphous $\mathrm{R}_{\text {Cullis }}{ }^{\mathrm{c}}$

$0,0,2.783$

1.033, 0.972, 5.406

$0.789,0.718,0.362$

anomalous $\mathrm{R}_{\text {Cullis }}{ }^{\mathrm{d}}$

0

0.882

1.012

0.463

0.259

0.967

Mean figure of merit (centric, acentric)

0.163, 0.195

Values in parentheses refer to the last resolution shell.

${ }^{\text {a }} \mathrm{R}_{\text {sym }}=\Sigma|\mathrm{I}-<\mathrm{I}>| / \Sigma \mathrm{I}$, where I is the observed intensity, and $<\mathrm{I}>$ is the average intensity obtained

${ }^{\mathrm{b}}$ Phasing power $=\left\langle\left|\mathrm{F}_{\mathrm{H}}\right|>/ \sigma(\mathrm{I} \Delta \mathrm{I})\right.$; the values given show the phasing power for isomorphous acentric, isomorphous centric, anomalous acentric reflections

${ }^{c}$ Isomorphous $\mathrm{R}_{\text {Cullis }}=\Sigma_{\text {hkl }} \Sigma \phi \mathrm{P}(\phi) \| \mathrm{F}_{\mathrm{P}}(\mathrm{obs})+\mathrm{F}_{\mathrm{H}}($ calc $)\left|-\mathrm{F}_{\mathrm{PH}}(\mathrm{obs})\right| / \Sigma_{\mathrm{hkl}}\left|\mathrm{F}_{\mathrm{PH}}(\mathrm{obs})-\mathrm{F}_{\mathrm{P}}(\mathrm{obs})\right|$, for centric reflections

${ }^{\mathrm{d}}$ Anomalous $\mathrm{R}_{\text {Cullis }}=\Sigma_{\text {hkl }} \Sigma \phi \mathrm{P}(\phi) \mid \Delta_{\text {ano }}(\mathrm{obs})-\Delta_{\text {ano }}($ calc $)\left|/ \Sigma_{\text {hkl }}\right| \Delta_{\text {ano }}($ obs $) \mid$ 


\section{Figure S1}

\section{a}

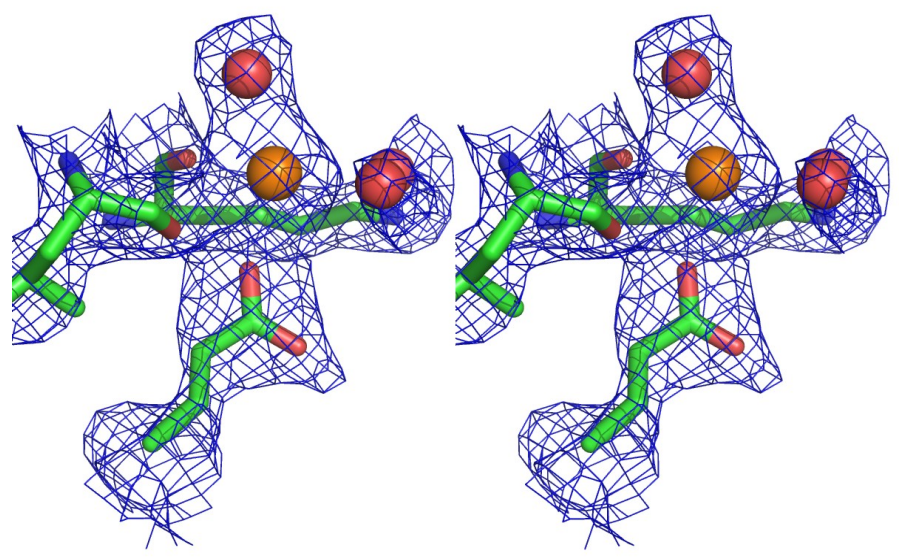

b

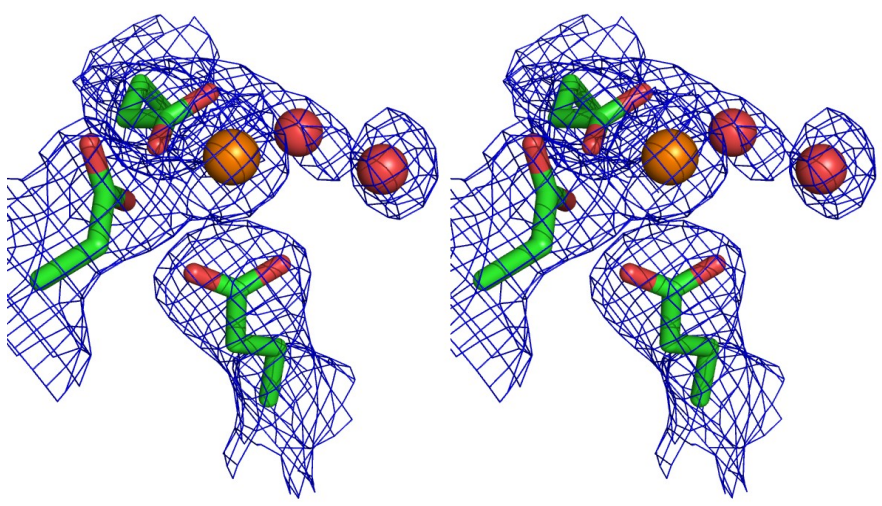

\section{Figure S1}

Calcium binding sites in $\alpha 1$-giardin: type III site in repeat I ( $\boldsymbol{a}$ ) and a novel site between repeats I and IV $(\boldsymbol{b})$. Stereo images of the two sites are shown with the final $2 \mathrm{~F}_{\mathrm{o}}-\mathrm{F}_{\mathrm{c}}$ density contoured at $1 \sigma$. Amino acids involved in coordination are drawn as stick models, and the oxygen atom of water molecules are shown as red spheres. Figure prepared with $\mathrm{PyMol}^{53}$. 


\section{Figure S2}

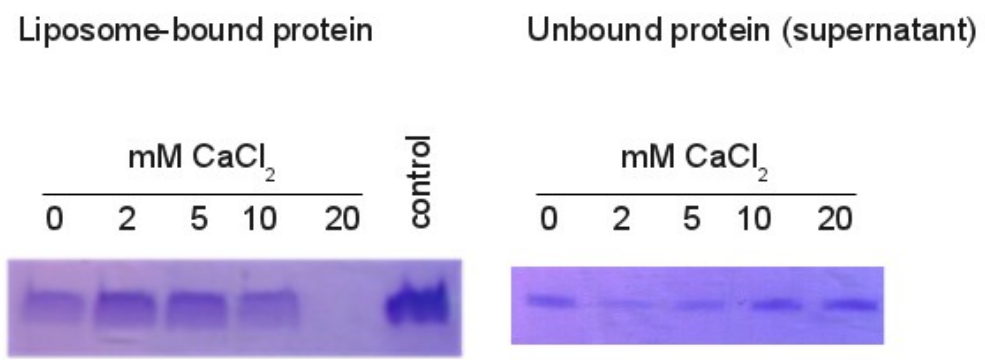

\section{Figure S2}

Representative SDS-PAGE analysis of the liposome co-pelleting assay using PS/PC (3:1) liposomes and calcium concentrations as indicated. After incubation with protein, the individual samples were centrifuged and the resulting pellets dissolved in $50 \mathrm{uL}$ of $10 \%$ SDS and subjected to SDS-PAGE. The same amount of protein loaded onto the samples was also dissolved in a total volume of $50 \mathrm{uL}$ $10 \%$ SDS and run as "control" (left gel image). Data from this step were used to construct the calcium-dependent membrane binding curves. Unbound protein was assessed by a separate SDSPAGE (right gel image). 


\section{Figure S3}

Heparin resin-bound protein (supernatant after EDTA wash)

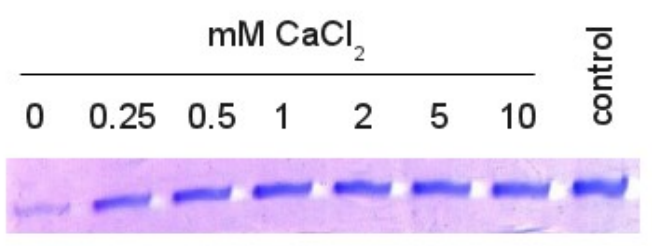

Unbound protein (supernatant after loading)

Irreversibly bound protein (resin after EDTA wash)

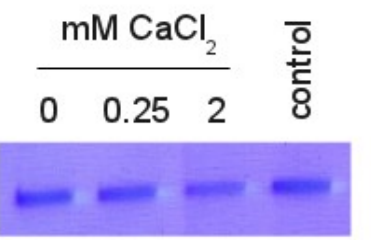

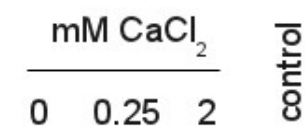

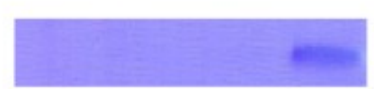

\section{Figure S3}

Representative SDS-PAGE analysis of the heparin binding assay using the calcium concentrations as indicated. After Loading of the protein, the resin suspension was centrifuged and the resulting supernatant analysed as "unbound protein" (middle SDS PAGE image). The resin was then washed with EDTA to release any protein bound in a calcium-dependent manner and centrifuged. The supernatant from this step was analysed as "bound protein" (left SDS PAGE) and the data used to construct the calcium-dependent heparin binding curve. The resin after EDTA wash was assessed by SDS-PAGE for any remaining protein ("irreversibly bound protein", right gel image). The control lanes show samples of the same amount of protein loaded onto the resin. 